\title{
Valproate-associated Hyperammonemic Encephalopathy
}

\author{
Jim Wadzinski, MD, Ronald Franks, MD, David Roane, PhD, and Max Bayard, MD
}

The use of valproic acid (VPA) (also known as Depakote, Depakene, and others) frequently results in elevated plasma ammonia. In some people, hyperammonemia may be clinically significant, resulting in hyperammonemic encephalopathy, which may be severe. Valproic acid-induced hyperammonemic encephalopathy may occur in people with normal liver function, despite normal doses and serum levels of VPA. We describe 2 cases of valproic acid-induced hyperammonemic encephalopathy in patients with supratherapeutic VPA levels, although the condition has been described in people with normal VPA levels. With the increasing indications and off-label uses of VPA, family physicians should be aware of this potential complication of VPA and check ammonia levels in patients taking VPA who present with alterations in mental status. Treatment with L-carnitine may be beneficial in reducing ammonia levels. ( $\mathrm{J}$ Am Board Fam Med 2007;20:499-502.)

Valproic acid (VPA) is effective in the treatment of seizure disorders, bipolar disorder, migraine headache prophylaxis, neuropathic pain, restless legs syndrome, dementia-related agitation, and social anxiety disorder, among other conditions. VPA has numerous drug interactions and toxicities; severe toxicities include hepatic damage, pancreatitis, teratogenicity, thrombocytopenia, and hyperammonemia. Here we depict 2 case reports of VPAinduced hyperammonemic encephalopathy (VHE), both occurring in patietns with no history of underlying liver disease. In one instance, the patient was able to function, but with significant cognitive limitations. In the second case, the patient was comatose. Both of the patients we describe also had supratherapeutic VPA levels, but VHE is a welldocumented potential complication of the use of VPA in the medical literature, and it may occur in people with normal VPA levels. ${ }^{1}$ Because of the

This article was externally peer reviewed.

Submitted 5 March 2007; revised 14 May 2007; accepted 16 May 2007.

From the Department of Family Medicine (JW, MB), the Department of Psychiatry (RF), and the Department of Pharmaceutical Sciences (DR), East Tennessee State University, Johnson City.

Funding: none.

Conflict of interest: none declared.

Corresponding author: Max Bayard, MD, East Tennessee State University, Johnson City Family Practice, 917 West Walnut, Johnson City, TN 37604 (E-mail: bayard@ mail.etsu.edu). wide spectrum of symptoms associated with VHE, physicians should consider hyperammonemia in the differential diagnosis of any patient taking VPA who shows changes in behavior, cognition, or orientation.

\section{Case Reports}

\section{Case 1}

A 51-year-old woman was transferred from a psychiatric hospital to an emergency department to evaluate the recent deterioration in her mental status. She had been admitted to the psychiatric hospital for an exacerbation of posttraumatic stress disorder and was placed on extended-release VPA at $1,000 \mathrm{mg}$ nightly. After 7 days of treatment she was no longer responsive to verbal stimuli. Her medical history was significant for migraine headaches, posttraumatic stress disorder, and major depression. Her medications included topiramate (for migraine prophylaxis), quetiapine, and VPA. In the emergency department, she was nonresponsive to verbal or painful stimuli; her vital signs were normal. Her pupils were equal at $5 \mathrm{~mm}$ and her ammonia level was $232 \mu \mathrm{mol} / \mathrm{L}(\mathrm{N}=10$ to 47$)$; her VPA level was $145 \mu \mathrm{g} / \mathrm{mL}(\mathrm{N}=50$ to 100$)$; and aspartate aminotransferase and alanine aminotransferase were normal at $17 \mathrm{IU} / \mathrm{L}$ and $19 \mathrm{IU} / \mathrm{L}$, respectively. The remainder of laboratory tests, including all liver tests, were normal. For the first 24 hours, the patient remained nonresponsive; her 
ammonia level dropped to $179 \mu \mathrm{mol} / \mathrm{L}$ with maintenance intravenous fluids and withdrawal of VPA. Beginning day 2, $50 \mathrm{mg} / \mathrm{kg} /$ day of Lcarnitine was administered once daily by nasogastric tube. The next morning, our patient's ammonia level was $56 \mu \mathrm{mol} / \mathrm{L}$, her VPA level was $54 \mu \mathrm{g} / \mathrm{mL}$, and she began to respond to verbal stimuli. Four days after admission from the emergency department, the patient returned to her baseline mental status. With the ammonia level still slightly elevated at $57 \mu \mathrm{mol} / \mathrm{L}$, she was discharged with instructions to discontinue VPA, topiramate, and to continue the L-carnitine for 10 days at $50 \mathrm{mg} / \mathrm{kg} /$ day.

\section{Case 2}

A 29-year-old woman came to the emergency department for evaluation of altered mental status. The patient complained of diminished short-term memory, confusion, disorientation, hypersomnia, and blurred vision for 2 to 3 weeks. She had arrived at work that day appearing intoxicated (evidenced by slurred speech, ataxia, and difficulty with concentration and memory). Her medical history was significant for obsessive-compulsive disorder and bipolar disorder. She denied any recent use of alcohol. Her prescribed medications included VPA $1,500 \mathrm{mg} /$ day, fluvoxamine $400 \mathrm{mg}$ twice a day, and clonazepam $2 \mathrm{mg}$ every night. The patient had been taking VPA for 5 months before this admission and had tolerated it well, except for some complaints of mild sedation. Seventeen days before this admission, the patient had increased her VPA from 1,000 to $1,500 \mathrm{mg} /$ day. In the emergency department, her vital signs were normal but her speech was slurred and her responses were sluggish. Examination was otherwise normal. A comprehensive metabolic panel (including liver tests) was normal. Her VPA level was $113 \mu \mathrm{g} / \mathrm{mL}(\mathrm{N}=50$ to $100)$ and her ammonia level was $182 \mu \mathrm{mol} / \mathrm{L}(\mathrm{N}=$ 10 to 47). VPA was discontinued, and the next day her VPA level was $112 \mu \mathrm{g} / \mathrm{mL}$ and her ammonia level was $80 \mu \mathrm{mol} / \mathrm{L}$. The day after that, our patient's VPA and ammonia levels were $38 \mathrm{mg} / \mathrm{mL}$ and $41 \mu \mathrm{mol} / \mathrm{L}$, respectively. She was discharged with improving mental status. As an outpatient, VPA was discontinued and replaced with topiramate and gabapentin, with good response and minimal adverse effects.

\section{Clinical Findings}

Patients with VHE present with varying degrees of cognitive and behavioral dysfunction. People may be asymptomatic. One case report described a young man with increased aggressiveness. ${ }^{2}$ Elderly people may present with worsening dementia. Others may be comatose. Seizure activity may increase, ${ }^{3}$ and deaths have been reported. ${ }^{4}$

Other anticonvulsants may potentiate the effects of VPA. Phenobarbital and phenytoin may increase ammonia levels in peole taking VPA. ${ }^{5}$ The addition of topiramate to VPA has precipitated VHE in previously asymptomatic people. ${ }^{6}$ Topiramate has been shown to inhibit the urea cycle and glutamine synthetase activity, both of which are likely mechanisms in the development of VHE. ${ }^{6}$

\section{Laboratory Findings}

Barring other underlying liver disease, patients with VHE may have no other laboratory derangements than an elevated serum ammonia. VPA levels may be normal and do not necessarily correlate with the degree of hyperammonemia or the severity of symptoms. Patients with a deficiency of ornithine transcarbamylase may have abnormal levels of various amino acids, including increased levels of gluatamine and alanine and low levels of citrulline, arginine, and carnitine. Electroencephalograms show generalized slowing and increased epileptiform discharges.

\section{Treatment}

The primary treatment for VHE is the withdrawal of VPA. Complete recovery generally occurs over a period of 1 day to a few days. Prolonged recovery times have been reported. ${ }^{1}$

L-carnitine supplementation has been shown to improve the symptoms of VPA related toxicities. VPA causes decreased serum levels of carnitine. ${ }^{7,8}$ Deficiency of carnitine results in diminished mitochondrial function, with inhibition of the urea cycle in the liver. ${ }^{9}$ In a retrospective chart review, administration of L-carnitine was associated with reduced mortality in patients with severe VPA-induced hepatotoxicity. ${ }^{10}$ L-carnitine has also been shown to be effective in reducing ammonia levels and in improving symptoms of hyperammonemia. ${ }^{11}$ It is generally safe ${ }^{12}$ and may be given orally or intravenously at a dose of 50 to $100 \mathrm{mg} / \mathrm{kg} /$ day. 


\section{Pathophysiology}

Although VHE is rare, VPA frequently causes a rise in serum ammonia levels, usually resulting in asymptomatic hyperammonemia. Several studies have shown elevated ammonia levels in a large percentage of asymptomatic patients on VPA. ${ }^{13}$ In one study, ammonia levels were measured in 55 patients taking VPA and 12 patients taking other anticonvulsants. Twenty-nine of the 55 patients receiving VPA had elevated ammonia levels, but none of the patients receiving other anticonvulsants had elevated levels. ${ }^{4}$ There are currently no specific recommendations for screening people for asymptomatic hyperammonemia, nor are there any known consequences.

The metabolism of ammonia occurs primarily through the urea cycle. Ammonia is a by-product of the conversion of amino acids to $\alpha$-ketoacids. In the liver, ammonia is converted to urea, which is then excreted in the urine. VPA inhibits the activity of carbamoyl phosphate synthetase I, the first enzymatic reaction in the urea cycle, thereby hindering the excretion of ammonia and raising plasma ammonia levels.

The relationship between VPA and carnitine and the subsequent development of hyperammonemia is not completely understood. VPA reduces levels of carnitine by 2 methods. Structurally, VPA is a short, branched-chain fatty acid. Carnitine is a carrier-type molecule required for the transport and oxidation of fatty acids in the mitochondria. VPA is believed to complex with carnitine in such a manner that the renal excretion of carnitine is enhanced. ${ }^{14}$ In addition, VPA, carnitine, and coenzyme A form poorly productive intracellular complexes that have 2 negative effects. The first is a reduction in fatty acid use for energy needs. Because cellular energy demands remain constant there is likely an increase in compensatory amino acid oxidation and a subsequent increase in the production of nitrogenous waste. The second effect is caused by the binding of VPA to coenzyme A, which is required for the formation of $\mathrm{N}$-acetylglutamate, a powerful allosteric activator of carbamoyl phosphate synthetase I and critical to the regulation of nitrogen flux toward its appropriate metabolism. ${ }^{15}$ Collectively, these actions of VPA provide the basis for the development of clinically significant hyperammonemia and offer an explanation for the palliative effect of administered L-carnitine.
The development of VHE in patients treated with VPA may unmask underlying heterozygous deficiency of ornithine transcarbamoylase, another enzyme in the urea cycle. ${ }^{16}$ This $\mathrm{X}$-linked disorder occurs in approximately 1 of 30,000 women. Laboratory findings suggestive of ornithine transcarbamoylase deficiency include elevated urine levels of orotic acid; elevated blood levels of ammonia, glutamine, and alanine; and low levels of citrulline. Most cases of VPA-induced hyperammonemia, however, occur in people without a known enzyme deficiency.

The mental status change associated with hyperammonemia is not fully understood. However, a likely mechanism is that hyperammonemia stimulates increased glutamine synthetase activity, causing increased production of glutamine in astrocytes. Cerebrospinalfluid and blood levels of glutamine may be elevated in conjunction with hyperammonemia. ${ }^{17}$ Glutamine in astrocytes causes an osmotic shift of fluid into the astrocytes, producing astrocyte swelling and cerebral edema. ${ }^{18}$

\section{Conclusion}

VHE is a potentially serious consequence of the use of VPA; physicians should consider this possible cause of changes in mental status in patients treated with VPA. Mental status changes in patients, with the addition of a second anticonvulsant (especially topirimate), should prompt consideration of hyperammonemia. Patients with VPA-induced hyperammonemia may be asymptomatic, may have behavioral changes, or may have marked deteriorations in their level of consciousness. Deaths have been reported. The primary therapy is withdrawal of VPA; L-carnitine supplementation may decrease ammonia levels and improve symptoms.

\section{References}

1. Gerstner T, Buesing D, Longin E, et al. Valproic acid induced encephalopathy-19 new cases in Germany from 1994 to 2003-a side effect associated to VPA-therapy not only in young children. Seizure 2006;15:443-8.

2. Yehya N, Saldarini CT, Koski ME, Davanzo P. Valproate-induced hyperammonemic encephalopathy. J Am Acad Child Adolesc Psychiatry 2004;43:926-7.

3. Marescaux C, Warter JM, Micheletti G, Rumbach L, Coquillat G, Kurtz D. Stuporous episodes during treatment with sodium valproate: report of seven cases. Epilepsia 1982;23:297-305.

4. Triggs WJ, Gilmore RL, Millington DS, Cibula J, 
Bunch TS, Harman E. Valproate-associated carnitine deficiency and malignant cerebral edema in the absence of hepatic failure. Int J Clin Pharmacol Ther 1997;35:353-6.

5. Murphy JV, Marquardt K. Asymptomatic hyperammonemia in patients receiving valproic acid. Arch Neurol 1982;39:591-2.

6. Hamer HM, Knake S, Schomburg U, Rosenow F. Valproate-induced hyperammonemic encephalopathy in the presence of topiramate. Neurology 2000; 54:230-2.

7. Laub MC, Paetzke-Brunner I, Jaeger G. Serum carnitine during valproic acid therapy. Epilepsia 1986; 27:559-62.

8. Ohtani Y, Endo F, Matsuda I. Carnitine deficiency and hyperammonemia associated with valproic acid therapy. J Pediatr 1982;101:782-5.

9. Raskind JY, El-Chaar GM. The role of carnitine supplementation during valproic acid therapy. Ann Pharmacother 2000;34:630-8.

10. Bohan TP, Helton E, McDonald I, et al. Effect of L-carnitine treatment for valproate-induced hepatotoxicity. Neurology 2001;56:1405-9.

11. Bohles H, Sewell AC, Wenzel D. The effect of carnitine supplementation in valproate-induced hyperammonaemia. Acta Paediatr 1996;85:446-9.
12. LoVecchio F, Shriki J, Samaddar R. L-carnitine was safely administered in the setting of valproate toxicity. Am J Emerg Med 2005;23:321-2.

13. Verrotti A, Trotta D, Morgese G, Chiarelli F. Valproate-induced hyperammonemic encephalopathy. Metab Brain Dis 2002;17:367-73.

14. Camina MF, Rozas I, Castro-Gago M, Paz JM, Alonso C, Rodriguez-Segade S. Alteration of renal carnitine metabolism by anticonvulsant treatment. Neurology 1991;41:1444-8.

15. Coude FX, Grimber G, Parvy P, Rabier D, Petit F. Inhibition of ureagenesis by valproate in rat hepatocytes. Role of N-acetylglutamate and acetyl-CoA. Biochem J 1983;216:233-6.

16. Oechsner M, Steen C, Sturenburg HJ, Kohlschutter A. Hyperammonaemic encephalopathy after initiation of valproate therapy in unrecognised ornithine transcarbamylase deficiency. J Neurol Neurosurg Psychiatry 1998;64:680-2.

17. Vossler DG, Wilensky AJ, Cawthon DF, et al. Serum and CSF glutamine levels in valproate-related hyperammonemic encephalopathy. Epilepsia 2002; 43:154-9.

18. Brusilow SW, Maestri NE. Urea cycle disorders: diagnosis, pathophysiology, and therapy. Adv Pediatr 1996;43:127-70. 